\title{
Modelación de la distribución espacial del muérdago (Santalales: Loranthaceae) en las áreas verdes de la delegación Tlalpan, México
}

\author{
Modeling the spatial distribution of true mistletoe (Santalales: Loranthaceae) \\ in the green areas of Tlalpan, Mexico Delegation
}

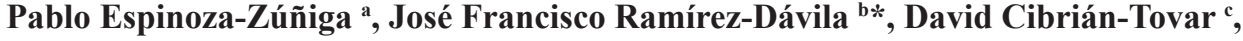 \\ Antonio Villanueva- Morales ${ }^{\mathrm{c}}$, Víctor David Cibrián-Llanderal d ${ }^{\mathrm{d}}$ Dulce Karen Figueroa- Figueroa ${ }^{\mathrm{e}}$, \\ Roberto Rivera- Martínez ${ }^{\mathrm{e}}$ \\ ${ }^{a}$ Universidad Autónoma Chapingo, Maestría en Ciencias en Ciencias Forestales, Texcoco, México. \\ *Autor de correspondencia: ${ }^{b}$ Universidad Autónoma del Estado de México, Toluca, \\ México, tel.: 7721952195, jframirezd@uaemex.mx \\ c Universidad Autónoma Chapingo, División de Ciencias Forestales, Texcoco, México. \\ d Instituto de Sanidad Forestal A.C, Texcoco, México. \\ ${ }^{e}$ Universidad Autónoma del Estado de México, Facultad de Ciencias Agrícolas, Toluca, México.
}

\begin{abstract}
SUMMARY
The objective of the present investigation was to model the spatial distribution of true mistletoe (Cladocolea loniceroides, Santalales: Loranthaceae) through the use of classical statistics, spatial statistics, geostatistics and generate maps showing the aggregations of this organism in the green areas of Tlalpan Delegation, Mexico City. A total of 40 sampling sites were evaluated, of which 12 presented mistletoe. With the use of Geostatistics, the spatial pattern of the mistletoe was modeled, experimental semivariograms were obtained and maps of aggregation of this pest and infested surface were generated. The results generated with the Spatial and Geostatistics presented an aggregate spatial structure. In contrast, the use of classical statistics did not yield congruent results and the spatial distribution of mistletoe could not be modeled with this method. The species of tree, surface of cups and general state of hosts, as well as plantation designs, seem to play an important role in the autocorrelation of the mistletoe-tree; with the distribution maps, the most infested areas could be determined and the different points of aggregation could be observed. The obtained information will allow better planning in the selection of the species to be planted, improving tree densities and coordinating management plans directed at the areas most affected by these pathogenic organisms.
\end{abstract}

Key words: geostatistics, true mistletoe, host, infestation, spatial statistics.

\section{RESUMEN}

El muérdago (Cladocolea loniceroides, Santales: Loranthaceae) es una planta hemiparásita que al impregnarse en el tallo de su hospedero, compite con él por agua y nutrimentos e impiden su desarrollo. Modelar la distribución espacial del muérdago en parques urbanos permite establecer mejores estrategias de control de esta hemiparásita. Por ello se hace uso de la estadística clásica, estadística espacial y geoestadística para generar mapas que muestren los diferentes focos de agregación de este organismo parásito en las áreas verdes de la Delegación Tlalpan, Ciudad de México. Se evaluaron 40 sitios de muestreo de los cuales 12 presentaron muérdago. Se modeló el patrón espacial del muérdago mediante geoestadística, se obtuvieron semivariogramas experimentales y se generaron mapas de agregación de esta plaga y superficie infestada. Los resultados generados con estadística espacial y geoestadística presentaron una estructura espacial agregada. Por el contrario, el uso de la estadística clásica no arrojó resultados congruentes y no se pudo modelar con este método la distribución espacial del muérdago. La especie de árbol, superficie de las copas y estado general de los hospederos, parecen jugar un papel importante en la auto correlación del muérdago-árbol; con los mapas se pudieron determinar las áreas mayormente infestadas y observar los diferentes puntos de agregación. La información obtenida permitirá mejores planeaciones en la selección de las especies a plantar, mejorar las densidades arbóreas y coordinar planes de manejo dirigidos sobre las áreas mayormente afectadas por estos organismos patógenos.

Palabras clave: geoestadística, muérdago verdadero, hospedero, infestación, estadística espacial. 


\section{INTRODUCCIÓN}

Los bosques urbanos son pulmones importantes para los entornos urbanizados, por lo tanto, su estudio y conservación son necesarios. En comparación con los árboles de áreas naturales, los árboles urbanos se desarrollan en condiciones más estresantes, lo que puede hacerlos más susceptibles a las infecciones por parásitos como el muérdago (Díaz et al. 2016). La presencia de las áreas verdes urbanas son importantes dentro de las ciudades ya que brindan muchos servicios ecosistémicos, incluyendo la captura de dióxido de carbono, mejor calidad del aire, reducción del ruido, la contaminación y un refugio para la fauna silvestre (Cantón et al. 2003).

Los muérdagos son plantas que se unen al tallo de los árboles, compiten con él por agua y nutrientes e impiden su desarrollo, cuando estos son abundantes sobre el árbol provocan la muerte del mismo (Alvarado y Saavedra 2007). El muérdago (Cladocolea loniceroides (Tiegh.) Kuijt, Santales: Loranthaceae) es una planta arbustiva que parasita los brotes aéreos de los hospederos; son organismos que pueden fotosintetizar, es decir, solo obtienen nutrientes minerales y agua del huésped (Mathiasen et al. 2008). Tienen un papel importante en el mantenimiento de la diversidad forestal al proporcionar recursos alimentarios claves para muchas especies animales debido a la disponibilidad de su fruto en épocas en que otros alimentos son escasos (Matula et al. 2015). Los efectos estudiados en el muérdago varían en función no solo de su capacidad de tomar agua y nutrientes del árbol hospedante, sino, también, dependen de las condiciones ambientales en las que se desarrolla el anfitrión, su tamaño, edad y densidad. En la ciudad de México se han realizado diversos estudios, en particular, se ha puesto especial atención en aquellas especies de árboles que han sido más susceptibles al ataque por muérdago (Alvarado y Saavedra 2007). El estudio del muérdago depende de la comprensión científica de la ecología de estos y el contexto del medio donde se desarrollan (Ramón et al. 2016); por lo tanto, es necesario tener en cuenta específicamente la ecología del muérdago, incluyendo la distribución espacial de la infección, los mecanismo de control relacionados y los procesos por los cuales se produce la propagación de la infestación (Rist et al. 2011).

Con el uso de nuevas herramientas, como métodos de análisis espacial, es posible describir la variación en la distribución espacial y la escala en las que se encuentran estos organismos (Aukema 2004). Entender el comportamiento espacial de las plantas parásitas (muérdago), puede brindar datos importantes para su manejo y de igual manera, proporcionar información sobre los mecanismos de dispersión de estas plantas parásitas (Andrew 2013). En un ambiente urbano, los árboles a menudo no se distribuyen al azar, sino que se plantan de tal manera que maximicen la estética y el sombreado, esto da lugar a una distribución con una alta dependencia espacial (Ricotta et al. 2001).
La geoestadística se define como un análisis estadístico de fenómenos naturales que se distribuyen de forma continua en el espacio o en el tiempo. Los estudios geoestadísticos proporcionan una medida más exacta de la dependencia espacial, ya que tienen en cuenta la naturaleza bidimensional de la distribución de los organismos a través de su localización espacial (Ramírez y Porcayo 2010). Se han realizado anteriormente investigaciones que determinan el comportamiento espacial de muérdago, los cuales han demostrado que la distribución espacial de este organismo en áreas naturales es agregada (Ward y Paton 2007). La distribución espacial del muérdago en áreas verdes urbanas no ha sido analizada ampliamente, por lo que resulta importante la aplicación de análisis espacial por índices de distancia (SADIE) para estudiar el comportamiento espacial de este parásito en ambientes urbanos. En la actualidad es casi nula la información sobre los patrones de distribución espacial del muérdago en los espacios verdes de las ciudades, por lo que es importante la aplicación de nuevas tecnologías que permitan ver la distribución espacial de estos organismos en el arbolado urbano.

El objetivo general de la presente investigación consiste en explorar los patrones de distribución espacial de las poblaciones del muérdago en las áreas verdes urbanas de la Delegación Tlalpan, Ciudad de México, mediante la aplicación de técnicas geoestadísticas, estadística espacial y estadística clásica, para contrastar la eficacia de estos métodos y elaborar mapas de densidad que muestren las áreas mayormente afectadas por estos organismos. La hipótesis de investigación supone que la distribución del muérdago es agregada y que las herramientas espaciales permiten modelar de mejor forma su distribución espacial.

\section{MÉTODOS}

La delegación Tlalpan, Ciudad de México se encuentra localizada en las coordenadas: Longitud Oeste: $99^{\circ} 13^{\text {' }}$ 30 " y Latitud Norte: $19^{\circ} 18^{\prime} 30^{\prime \prime}$ (INEGI 2014). Esta localidad cuenta con una superficie total de $307,84 \mathrm{~km}^{2}$, de los cuales $4,38 \mathrm{~km}^{2}$ son áreas verdes urbanas, concentrando el 4,93\% del total de la Ciudad de México (SEDEMA 2010).

Para realizar la modelación de la distribución espacial de muérdago en las áreas verdes de la Delegación Tlalpan, Ciudad de México se evaluaron 40 sitios de muestreo con una dimensión de $500 \mathrm{~m}^{2}$. Se levantó una muestra aleatoria de 20 árboles por cada sitio de muestreo, de los cuales se tomaron variables dasométricas como: diámetros, alturas, estrato arbóreo, condición física del árbol, especie del árbol, estado fitosanitario de los árboles, coordenadas espaciales y además se asignó una escala de nivel de infestación de la planta parasita (muérdago) que va de cero a seis; se generó una base de datos en el programa Excel® utilizando las coordenadas geográficas de los árboles y las escalas de infestación para su análisis correspondiente. 
Estadística clásica. Existen dos métodos generales para establecer la distribución espacial de los organismos binomial negativa y Poisson y los índices de dispersión (de dispersión y de Green). El modelo binomial negativo es probablemente la distribución más comúnmente utilizada para modelizar fenómenos agrupados, es decir, poblaciones contagiosas o agregadas (Silva et al. 2016). La binomial negativa está definida por dos parámetros: la media $\mathrm{m}$ $\mathrm{y}$ un exponente $\mathrm{k}$, o en términos del desarrollo de $(q-p)^{-\mathrm{k}}$, donde $\mathrm{p}=\mathrm{m} / \mathrm{k}$ y $\mathrm{q}-\mathrm{p}=1$. La probabilidad de encontrar $\mathrm{r}$ individuos en una muestra de una población de parámetros k y m será:

$$
P[\varepsilon=r]=(k+r-1)\left(\frac{1}{q}\right)^{k}\left(\frac{p}{q}\right)^{r}
$$

Como $\mathrm{m}=\mathrm{kp} \mathrm{y} \mathrm{s} \mathrm{s}^{2}=\mathrm{kp}(1+\mathrm{p})$, se deduce: para la población $\mathrm{s}^{2}=\mathrm{m}+\mathrm{m}^{2} / \mathrm{k}$ y para la muestra $\mathrm{s}^{2}=\bar{x}+x^{2} / \mathrm{k}$ (Guyon 2010).

La distribución que responde a una distribución aleatoria es la de Poisson, cuya principal característica es que la varianza es igual a la media: $\mathrm{s}^{2}=\mathrm{m}$. De aquí que se emplee como criterio de aleatoriedad de la distribución de frecuencias observadas a través de un muestreo, la realización entre la varianza y la media muestral es $\mathrm{s}^{2} / \bar{x}$ (Steel y Torrie 1985). Formalmente se puede expresar la distribución de Poisson de la siguiente manera: La probabilidad de encontrar un cierto número (r) de individuos en una muestra de una población con media $(\bar{x})$ y distribuida aleatoriamente es (Trujillo 2010):

$$
P[\varepsilon=r]=e^{-\bar{x}} \frac{\bar{x}^{r}}{r !}
$$

Multitud de índices han sido elaborados para establecer la distribución espacial de los organismos. Entre los índices más importantes se encuentran el índice de dispersión y el índice de Green (Rosas y Camarero 2004). El índice de dispersión se calcula con la siguiente fórmula:

$$
I D=\frac{s^{2}}{\bar{x}}
$$

Donde: $\bar{x}$ y $s^{2}$ son la media muestral y la varianza, respectivamente.

Si la muestra está en consonancia con una teórica serie de Poisson, se esperaría que este cociente fuera igual a 1. Por lo tanto, se puede comprobar, para una serie de datos cuando el número de muestras $(\mathrm{N})$ es inferior a 30 , si ID se desvía significativamente de 1 usando la prueba chi-cuadrado $\left(\mathrm{c}^{2}\right)$. Entonces, $\mathrm{Si}\left(s^{2}=\bar{x}\right)$ se acepta que se trata de una distribución de Poisson. Si $\left(s^{2}<\bar{x}\right)$ habrá una distribución regular o uniforme, finalmente, si $\left(s^{2}>\bar{x}\right)$ se estará ante una distribución en agregados o contagiosa.
Para tamaños de muestra grande $\left.\left(\mathrm{N}^{3} 30\right), \overline{(} \chi^{2}\right)$ tiende hacia una distribución normal, y se puede emplear la prueba estadística denominada $d$.

$$
d=\sqrt{\chi^{2}}-\sqrt{2(N-1)-1}
$$

Si $|d|<1,96$, se acepta que es una distribución al azar $(P$ $>0,05)$, si $|d|<-1,96$, se acepta que es una distribución regular o uniforme, y si $|d|>1,96$ se acepta que es una distribución agregada.

En cuanto al índice de Green, este se calcula con la siguiente fórmula:

$$
G I=\frac{\left(s^{2} / \bar{x}\right)-1}{N-1}=\frac{I C}{N-1}
$$

El GI varía entre -1/(N-1) (distribución uniforme) y 1 (distribución agregada). Si el valor GI es igual a 0 se tendrá una distribución al azar. Los valores entre 0 y 1 indican la fuerza de la agregación o contagio. Cuando el contagio es positivo, el índice de Green es independiente de $\mathrm{N}$ y de la media, lo cual son cualidades muy deseables para un índice de dispersión (Rosas y camarero 2004).

Estos métodos fueron utilizados en la presente investigación con el objetivo de realizar una comparación pertinente entre lo encontrado con la estadística no espacial y la estadística espacial. Para las distribuciones estadísticas se utilizó el programa MLP de máxima verosimilitud para ajustar los modelos a los datos obtenidos (Ross 1987). Las bondades de ajuste fueron examinadas con una prueba $\mathrm{c}^{2}$ (Ramírez y Porcayo 2010).

Estadística espacial SADIE. La base matemática de SADIE evalúa si un fenómeno es agregado, aleatorio o regular, para que se puedan evaluar dichos patrones se estima la distancia mínima en el espacio, $D$, requerida para obtener la regularidad, esto es, que los distintos valores de la variable estudiada alcancen el valor promedio de todas las posiciones en el espacio (Quero 2006). Los datos colectados en un sitio de muestreo prediseñado, se consideran un sistema de conteos de individuos, donde $i=1, . ., n$ unidades de muestreo, se conoce además, la posición bidimensional $(X i, Y i)$ de cada unidad de muestreo y su conteo asociado, $N_{i}$. La distancia para la regularidad, " $D$ ", es el valor mínimo de la distancia total que los individuos en la muestra pueden haberse movido, de una unidad de muestreo a otra. Si los conteos observados se permutan aleatoriamente entre las unidades de muestreo, de tal forma que la muestra que resulta sea un cambio o un reajuste simple de la original, entonces $P_{a}$ (probabilidad de agregación) representa la proporción de muestras seleccionadas al azar con distancia para la regularidad tan grande como el valor observado. Un valor de $P_{a}$ derivado de un número suficientemente grande de aleatorizaciones proporciona una prueba formal de aleatoriedad; la hipótesis nula de aleatoriedad espacial 
se puede rechazar, si $P_{a}<0,025$ (en favor de una hipótesis alternativa de agregación), o si $P_{a}>0,975$ (en favor de la alternativa de regularidad). Usualmente se dice que una muestra es agregada si $I_{a}>1$, la muestra es espacialmente aleatoria si $I_{a}=1$, y la muestra es regular si $I_{a}<1$ (Ramírez y Porcayo 2009). El programa utilizado en este trabajo para determinar los valores y las probabilidades de ambos índices fue el SADIE 1.22

Kriging. Se generó el semivariograma experimental con los valores de la escala de infestación del muérdago presente en las copas de los árboles muestreados en cada uno de los sitos de muestreo, calculado con la siguiente expresión (Moral 2004):

$$
y^{*}(h)=\frac{1}{2 N(h)} \sum_{i=1}^{N(h)}\left[z\left(x_{i}+h\right)-z\left(x_{i}\right)\right]^{2}
$$

Donde, $z=$ variable medida, $x_{i}=$ coordenada de una muestra, $x_{i}+h=$ coordenada de otra muestra a distancia $(\operatorname{lag}) h$ y $N(h)=$ número de pares de muestras $z\left(x_{i}\right)$ y $z\left(x_{i}\right.$ $+h)$.

La semivarianza esencialmente expresa la varianza promedio de pares de puntos a una distancia dada.

Para describir la auto correlación espacial de una variable cuantitativamente, se puede ajustar un modelo teórico al variograma empírico, para poder obtener los parámetros del modelo (efecto pepita, rango y meseta); el efecto pepita es el valor que representa la incertidumbre asociada a los datos experimentales o a la variabilidad espacial que pueda existir a una escala de muestreo menor que la efectuada (Sayad et al. 2017). El sill (umbral) es la varianza a la que el modelo del variograma alcanza (asintóticamente) un máximo; en muchas ocasione, el variograma empírico se estabiliza a una cierta distancia (Sayad et al. 2017). Si $z$ es estacionaria, $\gamma$ alcanza un valor límite constante llamado meseta $(\mathrm{C}+\mathrm{Co})$ que coincide con la varianza $\sigma^{2}$ de $z$. La distancia a la que se alcanza este valor se le denomina rango, este valor marca la zona de influencia en torno a un punto, más allá de la cual la auto correlación es nula.

Estimación de los parámetros del modelo de semivariograma. Una vez calculado el semivariograma experimental a cada sitio de muestreo se le ajusto un modelo teórico. Los modelos teóricos utilizados en la presente investigación fueron el esférico, exponencial y gaussiano, siguiendo los parámetros determinados por Samper y Carrera (1996). Para ajustar los semivariogramas experimentales a los semivariogramas de los muestreos en cada sitio se usó el programa Variowin 2.2 (Maldonado et al. 2016).

Validación del modelo teórico. Los modelos teóricos comúnmente usados para ajustar los semivariogramas experimentales son: esférico, gaussiano, exponencial, logarítmico, el de efecto pepita puro y el monómico; para la va- lidación de los parámetros (Co, efecto pepita, $\mathrm{C}$, meseta y a, rango o alcance) se van modificando hasta la obtención de validación cruzada adecuada (Maldonado et al. 2016). Estos estadísticos se mencionan a continuación:

A. Media de los errores de estimación. El MEE no debe ser significativamente diferente de 0 (prueba de $t$ ), en cuyo caso indicaría que el modelo del semivariograma permite el cálculo de estimaciones no sesgadas.

B. Error cuadrático medio (ECM). Un modelo de semivariograma se considera adecuado si, como regla práctica, el ECM es menor que la varianza de los valores muéstrales.

C. Error cuadrático medio adimensional (ECMA). La validez del modelo se satisface si ECMA está comprendido entre los valores $1 \pm 2(2 / \mathrm{N})^{0.5}$. Además se debe cumplir que la varianza de los errores de estimación debe ser $\leq$ que la varianza muestral (Samper y Carrera 1996).

Nivel de dependencia espacial. La determinación del grado de relación o nivel de dependencia espacial se obtuvo al dividir el efecto pepita entre la meseta y expresado en porcentaje su resultado. Si el resultado es menor que el $25 \%$ el nivel de dependencia espacial es alta, si se encuentra entre 26 y $75 \%$ el nivel de dependencia espacial es moderado y si es mayor del $76 \%$ el nivel de dependencia es bajo (Sayad et al. 2017).

Elaboración de mapas. Se realizó la interpolación a través del Krigeado ordinario, esta técnica permite la estimación insesgada de valores asociados a puntos que no fueron muestreados, las estimaciones obtenidas fueron representadas en forma de mapas para cada sitio de muestreo mediante el uso del programa Surfer 9.0. Con los mapas realizados, se calculó la superficie infestada, utilizando el método del Krigeado ordinario, se determinó el porcentaje de infestación cubierto por el muérdago en los sitios de muestreo; para la elaboración de los mapas se utilizó el programa Surfer 9.0 (Moral 2004).

\section{RESULTADOS}

Estado general del arbolado muestreado en la Delegación Tlalpan, Ciudad de México. Hay una diversidad de 34 especies arbóreas, siendo Fraxinus uhdei (Wenz.) Lingelsh. (Fresno) el más frecuente, con una presencia del 26,1\%. Del total de sitios muestreados el $62,5 \%$ se encuentran afectados por muérdago, mientras que el 37,5 \% no presenta ningún tipo de afectación. La especie de muérdago encontrada en los sitios de muestreo fue Cladocolea loniceroides (Tiegh.) Kuijt, con un 44,4 \% del arbolado infestado, le sigue Strthanthus interruptus (Kunth) G. Don $(5,7 \%)$ y Phoradendron velutinum (DC.) Olic. (9,7 \%), sin embargo, estos se encontraron en porcentajes muy ba- 
jos; mientras que el 37,5\% de los árboles muestreados no presentó infestación de este organismo. El arbolado de la Delegación Tlalpan también presentó afectaciones por insectos defoliadores en un 10,5\%. Con relación a los daños físicos el $58,9 \%$ se encuentran afectados por malas podas. El diámetro normal estimado fue de $29,5 \mathrm{~cm}$, con un valor mínimo de $10 \mathrm{~cm}$ y un máximo de $30 \mathrm{~m}$. Respecto a la altura promedio, se estimó en $18,3 \mathrm{~m}$, con un valor mínimo de $3 \mathrm{~m}$ y un máximo de $30 \mathrm{~m}$. Con relación a las copas de los árboles, el 36,1 \% correspondes al estrato arbóreo codominate. Por otro lado, de los árboles muestreados (800), el $61,8 \%$ era adulto, mientras que el $38,2 \%$ correspondió a la etapa juvenil.

El muérdago se encontró en cinco especies, sin embargo, el árbol más susceptible fue Populus tremuloides Michx., con un $100 \%$; después se encontró el Populus alba L. con un $82,4 \%$, seguido de Ulmus parvifolia Jacq. con un $50 \%$, Fraxinus uhdei con $25 \%$ y Schinus terebinthifolia Raddi con 11,1\%. En general los árboles que presentaban infestación de muérdago son aquellos árboles vigorosos de copas amplias y de buen tallo y posterior a la infestación estos árboles presentaban clorosis, tumores y hongos.

Estadística no espacial. En términos generales el índice de dispersión mostró que la distribución del muérdago es agregada, sin embargo, en los sitios 13 y 30 este índice mostró una distribución aleatoria. Con el índice de Green se confirmó la agregación encontrada con el índice de dispersión, los valores arrojados por este índice son bajos, por lo que se puede decir que la agregación del muérdago en los sitios de muestreo es leve (cuadro 1). Con relación a las distribuciones estadísticas los resultados muestran que en los sitios 1, 11, 13, 19, 24, 26, 29 30, 32 y 38 los datos se ajustaron a una binomial negativa (agregación), el sitio 30 se ajustó a una distribución de Poisson (distribución aleatoria). Los datos de los sitios 40 y 18 no se ajustaron a ningún tipo de distribución con la aplicación de estos índices.

Estadística espacial. Los resultados obtenidos con los índices $\left(I_{a,} J_{a}\right)$ evaluados con el SADIE se muestran en el cuadro 2. El índice $I_{a}$ toma el valor más alto en el sitio 11 , por el contrario el valor más bajo se encuentra en el sitio 19; en la mayoría de los sitios de muestreo este índice tomo un valor mayor a 1 , lo cual indica que el muérdago tiene una distribución espacial agregada.

Por su parte el índice $J_{a}$ su valor más alto se encontró en el sitio 32 y el valor más bajo se dio en el sitio 24. Los valores que tomo el índice $J_{a}$ fueron mayores a 1 , esto refuerza lo encontrado con el índice $I_{a}$ de que la distribución del muérdago es agregada. El valor del índice $J_{a}$ en los sitios de muestreo no fue significativamente superior a 1 , esto indica que la distribución espacial del muérdago se concentra en diferentes zonas de agregación (cuadro 2).

Análisis geoestadístico. Los modelos de semivariogramas ajustados a los datos del estudio fueron en su mayoría esféricos, sin embargo, también se ajustaron modelos gaussianos y exponenciales (cuadro 3). El efecto pepita para todos fueron valores cercanos a cero; este valor representa el error experimental. El nivel de dependencia espacial de las poblaciones de muérdago en todos los casos fue alta (cuadro 3). Los estadísticos de la validación cruzada permitieron la validación de los modelos ajustados, al hallarse

Cuadro 1. Índices de dispersión y distribuciones estadísticas del muérdago. Scatter rates and statistical distributions of true mistletoe.

\begin{tabular}{clccccc}
\hline & Sitio/Colonia & Índice de dispersión & Índice de Green & Poisson & Binomial negativa & $k$ \\
\hline 1 & Miguel Hidalgo & $3,54^{\mathrm{s}}$ & 0,012 & $\mathrm{NS}$ & $\mathrm{S}$ & 1,14 \\
11 & Tlalpan & $2,51^{\mathrm{s}}$ & 0,007 & $\mathrm{NS}$ & $\mathrm{S}$ & 2,21 \\
13 & Guadalupe & $1,47^{\text {ns }}$ & 0,002 & $\mathrm{NS}$ & $\mathrm{S}$ & 4,12 \\
18 & Ajusco & $2,08^{\mathrm{s}}$ & 0,003 & $\mathrm{NA}$ & $\mathrm{NA}$ & - \\
19 & Jardines de La Montaña & $3,49^{\mathrm{s}}$ & 0,009 & $\mathrm{NS}$ & $\mathrm{S}$ & 2,75 \\
24 & Glorieta de Vaqueritos & $2,87^{\mathrm{s}}$ & 0,008 & $\mathrm{NS}$ & $\mathrm{S}$ & 1,99 \\
26 & La Fama & $2,66^{\mathrm{s}}$ & 0,011 & $\mathrm{NS}$ & $\mathrm{S}$ & 2,63 \\
29 & San Miguel Ajusco & $2,35^{\mathrm{s}}$ & 0,006 & $\mathrm{NS}$ & $\mathrm{S}$ & 3,28 \\
30 & Nueva Oriental & $1,74^{\text {ns }}$ & 0,004 & $\mathrm{~S}$ & $\mathrm{~S}$ & 6,45 \\
32 & San Andrés Totoltepec & $3,07^{\mathrm{s}}$ & 0,005 & $\mathrm{NS}$ & $\mathrm{S}$ & 2,17 \\
38 & Fuentes del Pedregal & $2,91^{\mathrm{s}}$ & 0,010 & $\mathrm{NS}$ & $\mathrm{S}$ & 2,04 \\
40 & Parque Torriella & $2,63^{\mathrm{s}}$ & 0,002 & $\mathrm{NA}$ & $\mathrm{NA}$ & - \\
\hline
\end{tabular}

$\mathrm{S}=$ significativa; $\mathrm{NS}=$ no significativa; nivel de significación al $5 \%$; NA = no ajustado. 
en los rangos de aceptación matemática (cuadro 4). Los semivariogramas (modelos ajustados) elaborados para las poblaciones del muérdago en los sitios de muestreo se muestran en la figura 1 .

Elaboración de mapas de densidad (Krigeado). Con la elaboración de los mapas de densidad de las poblaciones de la planta parasita (muérdago) se pudo observar que la distribución espacial es en agregados (figura 2). Para representar el nivel de infestación del muérdago presente en las copas de los arboles $(1,2,3,4,5,6)$ en cada uno de los sitios de muestreo se utilizaron colores, el blanco indica que no hay presencia de este parasito y el rojo es el color que representa la escala de infestación más alta. En los mapas elaborados para cada uno de los sitos de muestreo se puede observar que hay diferentes focos de agregación de esta planta parásita; de igual forma con la representación de estos mapas se logró visualizar la distribución espacial en agregados de las poblaciones de muérdago, tal y como lo indicó el análisis geoestadístico y la estadística clásica.

Cuadro 2. Valores de los índices $\left(I_{a}, J_{a}\right)$ y sus probabilidades $P_{a}$ y $Q_{a}(\mathrm{~s}=$ significativa; $\mathrm{ns}=$ no significativa $)$. Values of indices $\left(I_{a^{\prime}} J_{a}\right)$ and their probabilities $P_{a}$ and $Q_{a}$.

\begin{tabular}{clccccc}
\hline & Sitio/Colonia & Sitio/Colonia & $I a$ & $P a$ & $J a$ & $Q a$ \\
\hline 1 & Miguel Hidalgo & 1 & 1,62 & $0,011^{\mathrm{s}}$ & 1,20 & $0,19^{\text {ns }}$ \\
11 & Tlalpan & 11 & 1,73 & $0,008^{\mathrm{s}}$ & 1,09 & $0,21^{\text {ns }}$ \\
13 & Guadalupe & 13 & 1,56 & $0,010^{\mathrm{s}}$ & 1,17 & $0,14^{\text {ns }}$ \\
18 & Ajusco & 18 & 1,67 & $0,015^{\mathrm{s}}$ & 1,13 & $0,26^{\text {ns }}$ \\
19 & Jardines de La Montaña & 19 & 1,44 & $0,009^{\mathrm{s}}$ & 1,16 & $0,17^{\text {ns }}$ \\
24 & Glorieta de Vaqueritos & 24 & 1,58 & $0,012^{\mathrm{s}}$ & 1,08 & $0,13^{\text {ns }}$ \\
26 & La Fama & 26 & 1,70 & $0,007^{\mathrm{s}}$ & 1,14 & $0,25^{\text {ns }}$ \\
29 & San Miguel Ajusco & 29 & 1,55 & $0,012^{\mathrm{s}}$ & 1,10 & $0,16^{\text {ns }}$ \\
30 & Nueva Oriental & 30 & 1,49 & $0,008^{\mathrm{s}}$ & 1,12 & $0,22^{\text {ns }}$ \\
32 & San Andrés Totoltepec & 32 & 1,53 & $0,014^{\mathrm{s}}$ & 1,21 & $0,15^{\text {ns }}$ \\
38 & Fuentes del Pedregal & 38 & 1,64 & $0,009^{\mathrm{s}}$ & 1,11 & $0,20^{\text {ns }}$ \\
40 & Parque Torriella & 40 & 1,51 & $0,013^{\mathrm{s}}$ & 1,15 & $0,18^{\text {ns }}$ \\
\hline
\end{tabular}

Cuadro 3. Parámetros de los modelos teóricos ajustados a los semivariogramas de muérdago por sitios de muestreo.

Parameters of theoretical models adjusted to true mistletoe semivariograms by sampling sites.

\begin{tabular}{llcccccc}
\hline & Sitio/Colonia & Varianza & Modelo & Pepita & Rango & Meseta & Pepita/ Meseta (\%) \\
\hline 1 & Miguel Hidalgo & 0,47 & Esférico & 0 & 10,40 & 0,19 & 0 \\
11 & Tlalpan & 0,67 & Gaussiano & 0 & 5,76 & 0,22 & 0 \\
13 & Guadalupe & 0,14 & Esférico & 0,008 & 10,36 & 0,10 & 8 \\
18 & Ajusco & 0,65 & Esférico & 0,045 & 11,90 & 0,23 & 19 \\
19 & Jardines de La Montaña & 0,40 & Esférico & 0 & 10,22 & 0,11 & 0 \\
24 & Glorieta de Vaqueritos & 0,40 & Exponencial & 0 & 11,88 & 0,13 & 0 \\
26 & La Fama & 0,58 & Esférico & 0 & 11,50 & 0,27 & 0 \\
29 & San Miguel Ajusco & 1,33 & Gaussiano & 0 & 6,50 & 1,18 & 0 \\
30 & Nueva Oriental & 0,80 & Esférico & 0 & 10,80 & 0,45 & 0 \\
32 & San Andrés Totoltepec & 0,82 & Esférico & 0,042 & 10,80 & 0,37 & 11 \\
38 & Fuentes del Pedregal & 1,95 & Gaussiano & 0 & 7,36 & 2,76 & 0 \\
40 & Parque Torriella & 1,21 & Gaussiano & 0 & 6,4 & 1,06 & 0 \\
\hline
\end{tabular}


Cuadro 4. Valores de los estadísticos de la validación cruzada.

Statistical values of cross validation.

\begin{tabular}{llcccccc}
\hline \multicolumn{1}{c}{ Sitio/Colonia } & $\begin{array}{c}\text { Tamaño de la } \\
\text { muestra }\end{array}$ & $\begin{array}{c}\text { Varianza } \\
\text { muestral }\end{array}$ & MEE & $\begin{array}{c}\text { Varianza de } \\
\text { los errores }\end{array}$ & ECM & ECMA \\
\hline 1 & Miguel Hidalgo & 20 & 0,47 & $0,06^{\text {ns }}$ & 0,22 & 0,14 & 1,08 \\
11 & Tlalpan & 20 & 0,67 & $0,13^{\text {ns }}$ & 0,46 & 0,10 & 1,13 \\
13 & Guadalupe & 20 & 0,14 & $0,08^{\text {ns }}$ & 0,10 & 0,08 & 1,10 \\
18 & Ajusco & 20 & 0,65 & $0,10^{\text {ns }}$ & 0,41 & 0,13 & 1,09 \\
19 & Jardines de La Montaña & 20 & 0,40 & $0,15^{\text {ns }}$ & 0,25 & 0,11 & 1,11 \\
24 & Glorieta de Vaqueritos & 20 & 0,40 & $0,09^{\text {ns }}$ & 0,19 & 0,09 & 1,15 \\
26 & La Fama & 20 & 0,58 & $0,14^{\text {ns }}$ & 0,50 & 0,06 & 1,07 \\
29 & San Miguel Ajusco & 20 & 1,33 & $0,07^{\text {ns }}$ & 1,14 & 0,12 & 1,12 \\
30 & Nueva Oriental & 20 & 0,80 & $0,11^{\text {ns }}$ & 0,67 & 0,07 & 1,14 \\
32 & San Andrés Totoltepec & 20 & 0,82 & $0,12^{\text {ns }}$ & 0,69 & 0,11 & 1,10 \\
38 & Fuentes del Pedregal & 20 & 1,95 & $0,09^{\text {ns }}$ & 1,52 & 0,10 & 1,09 \\
40 & Parque Torriella & 20 & 1,21 & $0,10^{\text {ns }}$ & 1,03 & 0,09 & 1,12 \\
\hline
\end{tabular}

$\mathrm{MEE}=$ media de los errores de estimación $; \mathrm{ECM}=$ error cuadrático medio; $\mathrm{ECMA}=$ error cuadrático medio adimensional

Superficie infestada. A partir de los mapas elaborados a través del Krigeado se pudo observar que hubo sitios de muestreo que presentaron una superficie infestada mayor al $50 \%$ (cuadro 5), el sitio 38 presentó el mayor porcentaje de infestación ( $88 \%$ ) de sus árboles y, por el contrario, el sitio 13 presentó el valor menor, ya que el $79 \%$ de los árboles de este sitio no presentó muérdago.

Cuadro 5. Porcentaje de superficie infestada y no infestada de muérdago por sitio de muestreo en la delegación Tlalpan. sampling site.

Distribution maps of true mistletoe, based on Krigeado per

\begin{tabular}{clcc}
\hline & Sitio/Colonia & \% infestado & \% no infestado \\
\hline 1 & Miguel Hidalgo & 79 & 21 \\
11 & Tlalpan & 81 & 19 \\
13 & Guadalupe & 21 & 79 \\
18 & Ajusco & 43 & 57 \\
19 & Jardines de La Montaña & 41 & 59 \\
24 & Glorieta de Vaqueritos & 45 & 55 \\
26 & La Fama & 42 & 58 \\
29 & San Miguel Ajusco & 30 & 70 \\
30 & Nueva Oriental & 75 & 25 \\
32 & San Andrés Totoltepec & 56 & 44 \\
38 & Fuentes del Pedregal & 81 & 12 \\
40 & Parque Torriella & 61 & 39 \\
\hline
\end{tabular}

\section{DISCUSIÓN}

La especie de árbol más frecuente en la zona de estudio fue Fraxinus uhdei con un 26,1\%, sin embargo, la población de estos árboles no fue la más susceptible al contagio de muérdago; las especies más afectadas por este organismo fueron Populus tremuloides, con un $100 \%$, después se encuentra Populus alba con un 82,4 \%, seguido de Ulmus parvifolia con un $50 \%$. El muérdago encontrado en los sitios de muestreo fue Cladocolea loniceroides con un 44,4 \% del arbolado infestado Yunuen et al. (2015) registraron la presencia de plantas parasitas del género Cladocolea y Struthanthus, siendo el Populus tremuloides la más afectada por estos muérdagos. Por otro lado, Arriola et al. (2012) encontraron Cladocolea loniceroides, Phoradendron velutinum y Struthanthus interruptus en siete delegaciones de la Ciudad de México sobre 17 hospederas pertenecientes a 10 familias botánicas. La presencia de insectos descortezadores, daños físicos y malas podas condicionan el estado del arbolado de la delegación, ya que debilitan la fisiología de estos, volviéndolos más vulnerables al ataque e infestación por estas plantas parásitas. Yunuen et al. (2015) Identificaron que dentro de los principales problemas del arbolado urbano de la Ciudad de México está el vandalismo, presencia de insectos chupadores, la limitación de espacio para el desarrollo de las raíces, plantaciones inadecuadas y traslape de doseles (copas). Esto último provoca que los árboles no reciban la luz del sol, limitando su fotosíntesis y otras funciones vitales y favoreciendo su debilitamiento. El huésped y las condiciones generales del hábitat, pueden ser factores que influyen en la distribución espacial de los muérdagos (Sayad et al. 2017). 


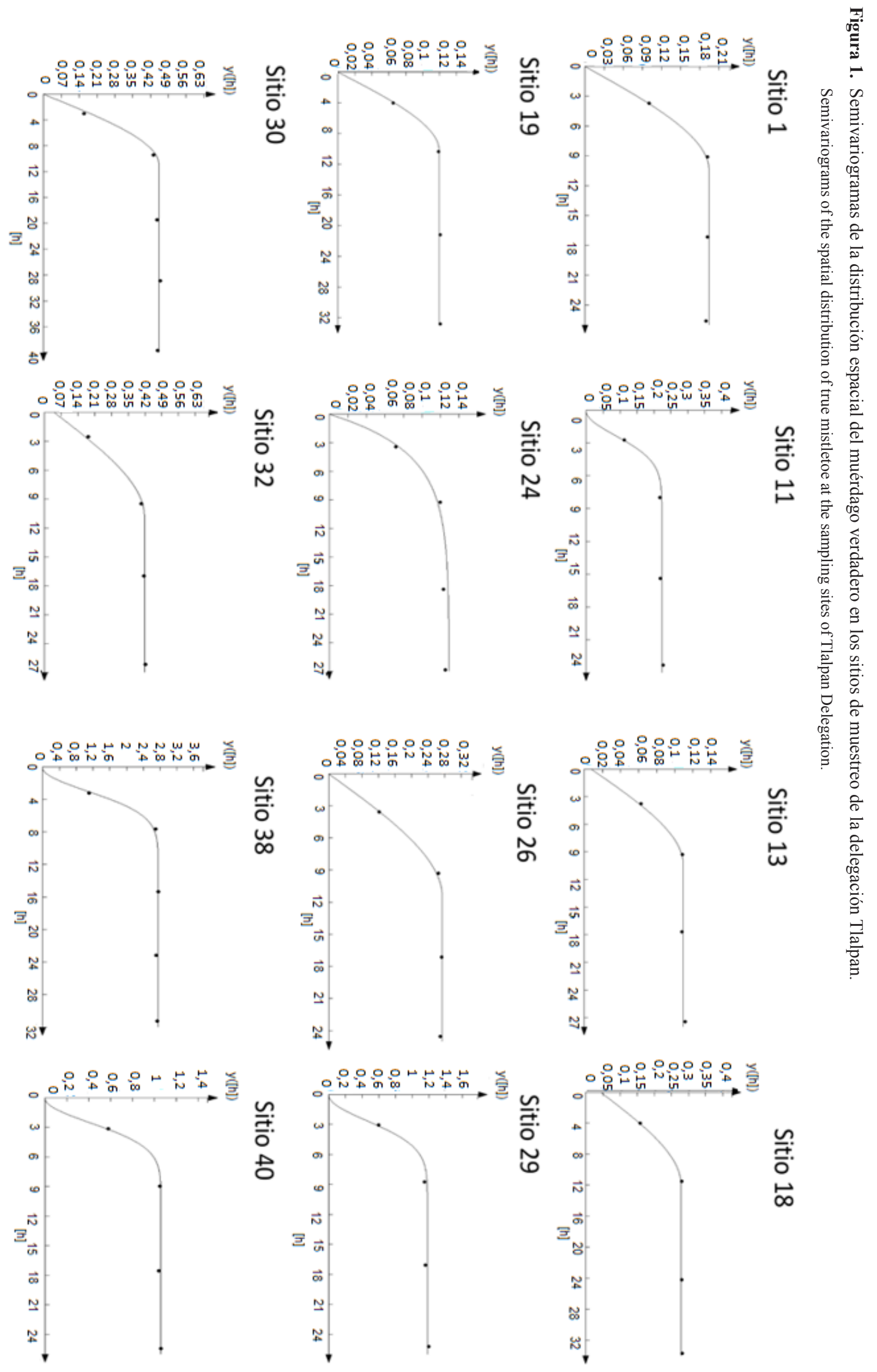


Los resultados obtenidos con los índices de dispersión y Green, mostraron una estructura espacial agregada, sin embargo, con la aplicación de estos índices se denotaron irregularidades, ya que en algunos casos se ajustaban a una distribución agregada y también a una aleatoria. En un estudio realizado por (Ramírez y Porcayo 2010), donde aplicaron estos métodos para determinar el patrón espacial de muérdago, encontraron que el índice de Green y de dispersión muestran una agregación en general de estos organismos; sin embargo, señalan ciertas incongruencias en la determinación de esa distribución. Estos autores indican en su estudio que el índice de Green es el más adecuado de los índices de dispersión, ya que es independiente del número de individuos y del valor medio de su abundancia, pero no es independiente del tamaño de la muestra. Un incon- veniente del índice de Green es que no tiene una varianza conocida, lo que impide calcular sus límites de significancia, esto aunado a que no incluye datos geoespaciales, por lo que no es posible indicar donde se encuentran los agregados. Probablemente esta desventaja es responsable del bajo uso de este índice para detectar patrones (Malhado y Petrere 2004). Con respecto a las distribuciones estadísticas, la mayoría de los sitios de muestreo presentó un ajuste a una binomial negativa (agregación), excepto el sitio 30, que se ajustó a una distribución de Poisson (distribución aleatoria) y los sitios 18 y 40, a los que no se les pudo ajustar ninguna distribución. En los sitios en los que se ajustó la binomial negativa, el valor de $k$ osciló entre 1,12 y 4,12, observándose los mayores valores (mayores grados de agregación) en los sitios 1 y 24 . El índice $k$ es un buen
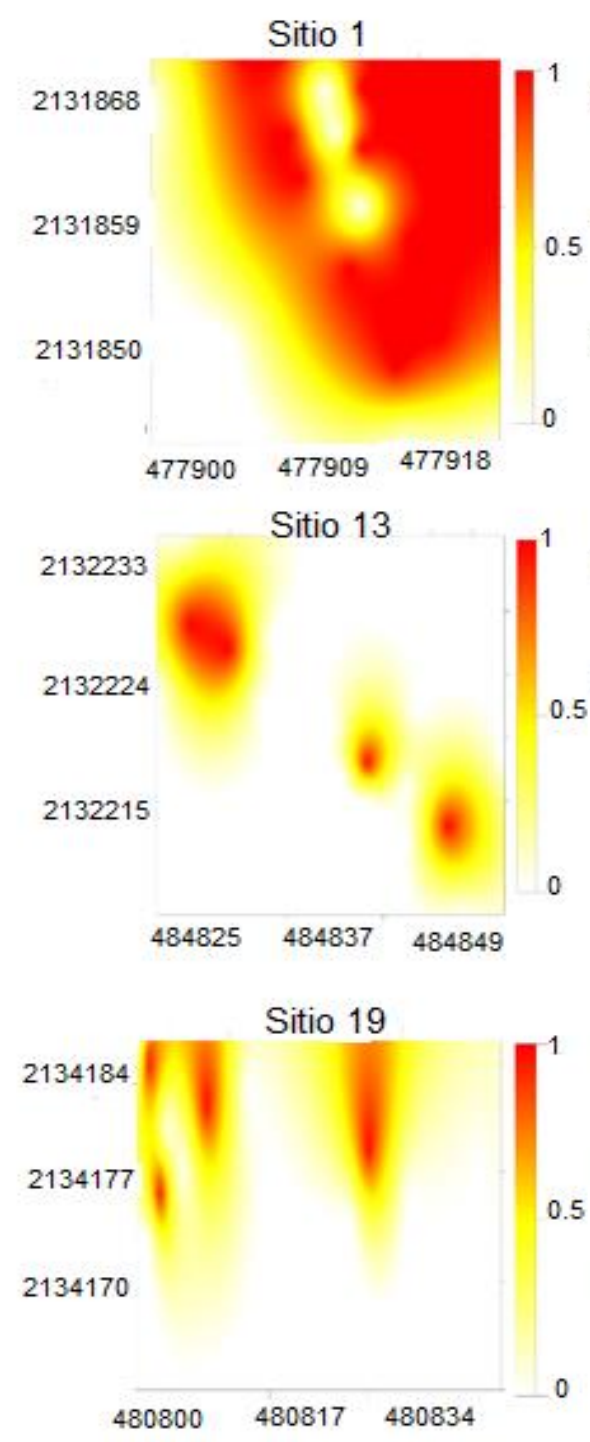

\section{Sitio 11}

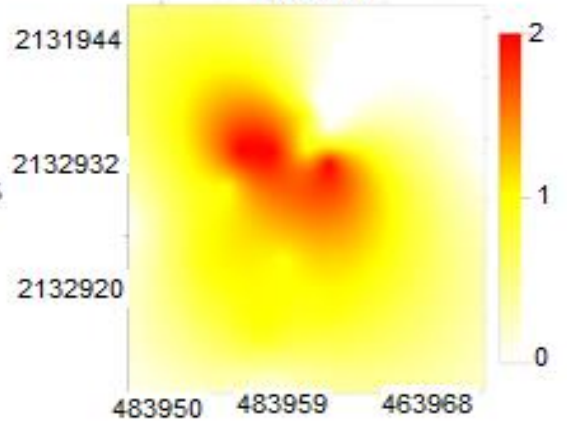

Sitio 18

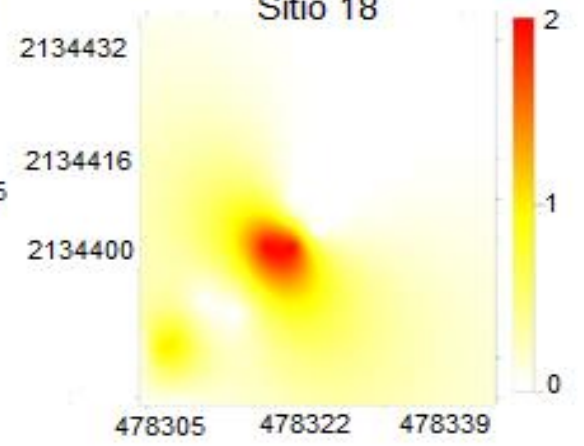

Sitio 24

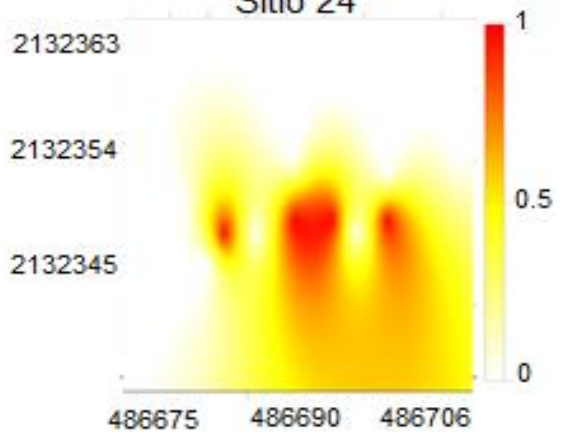

Continúa

Figura 2. Mapas de distribución del muérdago verdadero, en base al Krigeado por sitio de muestreo.

Distribution maps of true mistletoe, based on Krigeado per sampling site. 


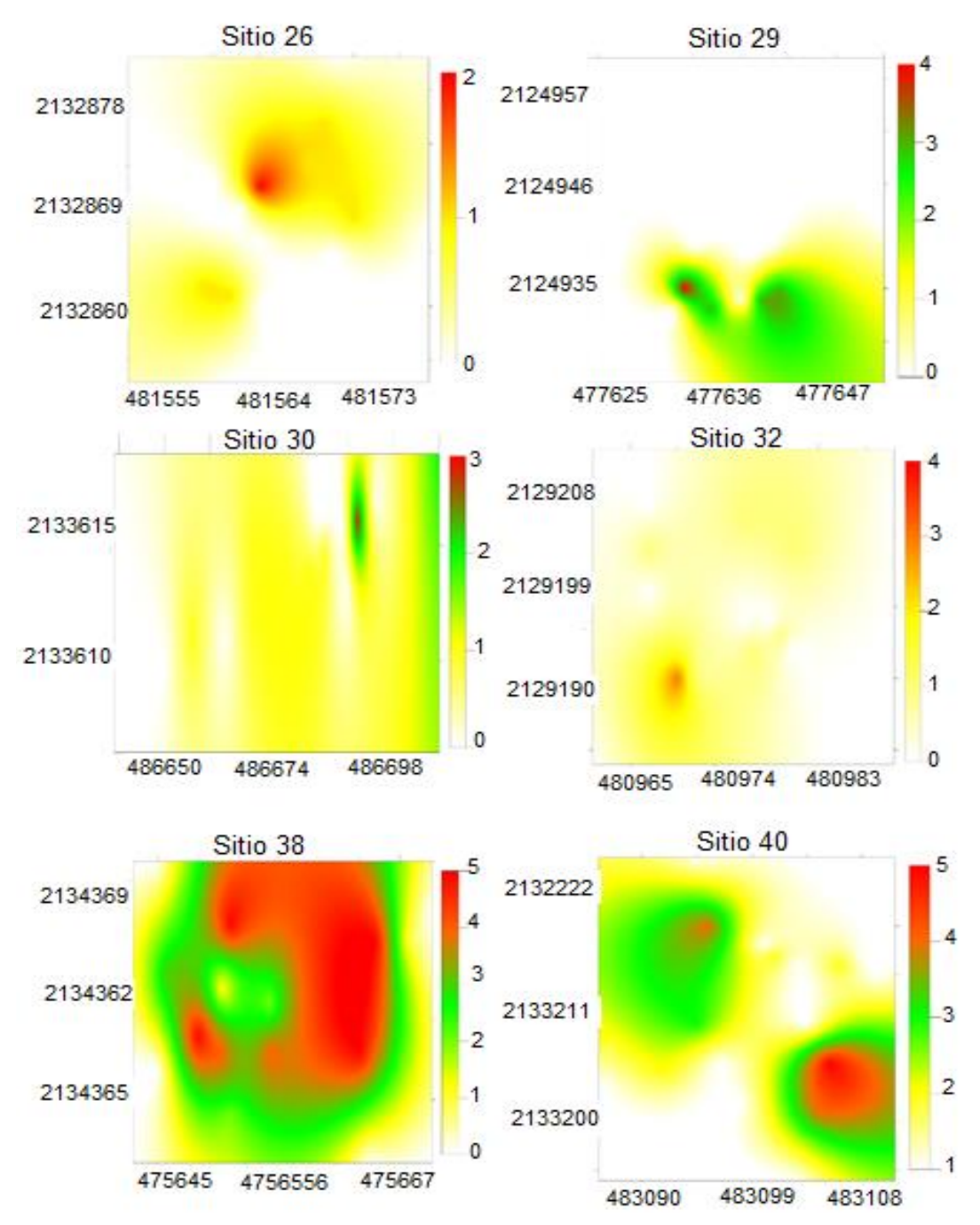

indicativo cuando el tamaño y el número de unidades de muestreo por muestra son los mismos, porque este índice es a menudo influenciado por el tamaño de las unidades de muestreo; este parámetro es una medida inversa del grado de agregación y valores negativos indican distribución regular o uniforme, los valores positivos cercanos a 0 indican una distribución agregada y valores superiores a 8 indican una disposición al azar (Melo et al. 2006).

La estadística clásica presentó ciertas limitaciones, ya que no cuenta con la naturaleza bidimensional de los datos por lo que no es posible saber dónde se ubican los centros de agregación por lo que no describe de forma clara y contundente el patrón espacial del muérdago; en muchos casos los resultados no son claros y en otros casos los datos no se ajustaron ningún tipo de distribución. Otro factor limitante del uso de la estadística clásica es que no toma en cuenta los datos bidimensionales de las poblaciones estudiadas, por lo que no se pudieron elaborar mapas de densidad del muérdago y ver en donde se encontraba la agregación.
Los resultados encontrados con los índices $\left(I_{a} \mathrm{y} J_{a}\right)$ señalan una agregación en general del muérdago. Ramírez y Porcayo (2009) encontraron una disposición espacial agregada de muérdago en un ambiente natural usando estos índices. Con esto se puede decir que el muérdago toma un patrón de distribución agregada tanto en áreas verdes urbanas como en bosques naturales. En el estudio gracias al índice $J_{a}$ se encontró que existen varios centros de agregación distribuidos en los 16 sitios de muestreo evaluados. Dávila et al. (2013) señalan que este índice, al no tener un valor significativamente superior a 1 , muestra una distribución agregada y que, además de esto, dicha agregación se concentra en diferentes puntos del área estudiada. Estas agregaciones se corroboraron con los mapas elaborados mediante el Krigeado (figura 2) (Maldonado et al. 2016).

El uso de la geoestadística permitió modelar la estructura espacial del muérdago, se ajustaron modelos esféricos en la mayoría de los sitios de muestreo, con un efecto pepita equivalente a cero. Los resultados encontrados mediante este método determinaron la existencia de una 
estructura espacial agregada. Una distribución agregada puede indicar que es más probable que el vector o agente dispersante disemine el parásito a cortas distancias, en lugar de a huéspedes más lejanos (Byamukama et al. 2011). Los niveles de dependencia espacial fueron altos para todos los casos, esto quiere decir que el muérdago tiene una fuerte dependencia espacial; Se considera que una variable tiene una fuerte dependencia espacial si el valor es menor que $25 \%$ y una dependencia espacial moderada si el valor esta entre el 25 y $75 \%$, de lo contrario, la variable tiene una débil dependencia espacial. Una dependencia espacial alta sugiere que la presencia de muérdago tendrá una mayor presencia en el tiempo en el lugar observado, determinando nichos ecológicos estables (Davila et al. 2013). Las distancias indicadas por el rango permiten inferir que hay altas probabilidades de que los árboles situados dentro de ese rango en una zona afectada sean infectados en un periodo de tiempo relativamente corto (Ramírez y Porcayo 2010). Este valor podría estar relacionado principalmente con el comportamiento de los vectores principales que son las aves, ya que primero visitan a los árboles más cercanos, infectados o no, lo que promueve una mayor transmisión de semillas de muérdago hacia estos (López et al. 2002). Para este estudio, el rango osciló entre 5,76 y $11,90 \mathrm{~m}$; sería interesante en estudios posteriores evaluar el comportamiento de las aves que cohabitan en las áreas verdes urbanas de la Ciudad de México, así como, evaluar la auto correlación espacial entre el muérdago y la densidad de árboles presentes, otro aspecto importante a considerar si el tipo de suelo juega un papel importante en la distribución espacial de esta planta parasita. Con la geoestadística se elaboraron mapas de densidad mediante la técnica del Krigeado simple, se pudieron determinar las áreas potencialmente infestadas y no infestadas por el muérdago, con la información generada con los mapas se pueden hacer planes de manejo de estos organismos y de esa forma optimizar los gastos económicos para su manejo y control.

\section{CONCLUSIONES}

Los métodos de estadística clásica (distribuciones estadísticas e índices de dispersión) muestran de forma general una estructura espacial agregada del muérdago; sin embargo, los resultados obtenidos denotan ciertas incongruencias y no dan una idea clara sobre la localización espacial de estos organismos. Por otro lado, con este método no es posible la elaboración de mapas de densidad que muestren los diferentes focos de agregación de esta planta parásita en las áreas estudiadas.

El uso de herramientas espaciales como el SADIE y la geoestadística permiten de forma pertinente modelar la distribución espacial de las poblaciones de muérdago. La información obtenida mediante el uso de métodos geoestadísticos indica la existencia de una estructura espacial agregada con un alto nivel de dependencia espacial. Los mapas elaborados mediante la técnica del Krigeado muestran los diferentes focos de agregación y manifiestan ser de gran utilidad en el análisis de la distribución de estas plantas parásitas. La identificación de áreas con porcentajes variables de infestación permitirá operar un control dirigido de la plaga en las zonas requeridas y reducir los gastos económicos para su manejo. La información recabada en este trabajo permitirá percatarse de la dimensión del problema que representa el muérdago para las áreas verdes de la zona y ayudará en gran medida a la propuesta, desarrollo y puesta en marcha de un programa de manejo para este problema fitosanitario preciso, adecuado y eficaz para reducir las poblaciones de esta planta hemiparásita.

\section{AGRADECIMIENTOS}

Al Dr. David Cibrián Tovar por el apoyo brindado para la realización del estudio. Al CONACyT por el financiamiento proporcionado para la realización de estudios de maestría. Al Instituto de Sanidad Forestal y, en especial, al Ing. Jesús Morales Bautista por el apoyo otorgado para el levantamiento de datos.

\section{REFERENCIAS}

Alvarado RD, L Saavedra. 2007. El género Cladocolea (Loranthaceae) en México: Muérdago verdadero o injerto. Revista Chapingo. Serie Ciencias Forestales y del Ambiente 11(1): 5-9.

Andrew VG. 2013. Spatial distribution of eastern mistletoe (Phoradendron leucarpum, Viscaceae) in an urban environment. Journal of Alabama Academy of Science 84: 155-167.

Arriola VJ, E Velasco, T Hernández, A González, ME Romero. 2012. True Mistletoes of the Trees of Mexico City. Revista Mexicana de Ciencias Forestales 4(19): 34-45. DOI: $\underline{10.29298 / \text { rmcf.v4i19.377 }}$

Aukema JE. 2004. Distribution and dispersal of desert mistletoe is scale-dependent, hierarchically nested. Ecography 27(2): 137-144.

Byamukama E, AE Robertson, FWJ Nutter. 2011. Quantifying the Within-Field Temporal and Spatial Dynamics of Bean pod mottle virus in Soybean. Plant Disease 95(2): 126-136. DOI: 10.1094/PDIS-07-09-0469

Cantón MA, C De Rosa, H Kasperidus. 2003. Sustentabilidad del bosque urbano en el área metropolitana de la ciudad de Mendoza. Análisis y diagnóstico de la condición de las arboledas. Avances en Energías Renovables y Medio Ambiente 7(1): 29-34.

Díaz LM, Z Cano, ME Queijeiro. 2016. Mistletoe infection in an urban forest in Mexico City. Urban Forestry \& Urban Greening 17: 126-134. DOI: 10.1016/j.ufug.2016.04.004

López L, F Ornelas, G García, J Franco. 2002. Mistletoe infection of trees located at fragmented forest edges in the cloud forests of Central Veracruz, Mexico. Forest Ecology and Management 164(1-3): 293-302. DOI: $10.1016 / \mathrm{S} 0378-$ $\underline{1127(01) 00624-7}$

Maldonado ZF, JF Ramírez, AV Lara. 2016. Distribución espacial de trips en aguacate en Coatepec Harinas, Estado de México. Revista Mexicana de Ciencias Agricolas 7(4): 845-856. 
Malhado CM, M Petrere. 2004. Behaviour of dispersion indices in pattern detection of a population of angico, Anadenanthera peregrina (Leguminosae). Brazilian Journal of Biology 64(2): 243-249.

Mathiasen RL, DC Shaw, DL Nickrent, D Watson. 2008. Mistletoes: Pathology, Systematics, Ecology, and Management. Plant Disease 92(7): 988-1006.

Matula R, M Svátek, M Pálková, D Volařík, T Vrška. 2015. Mistletoe infection in an oak forest is influenced by competition and host size. PloS one 10(5): 1-11. DOI: 10.1371/journal. pone. 0127055

Melo EP, MG De Fernandes, PE Degrande, RMA Cessa, L Jorge. 2006. Distribuição espacial de plantas infestadas por Spodoptera frugiperda (J. E. Smith) (Lepidoptera : Noctuidae) na cultura do milho. Neotropical Entomology 35(5): 689697. DOI: $10.1590 / \mathrm{S} 1519-566 \mathrm{X} 2006000500018$

Moral FL. 2004. Aplicación de la geoestadística en las ciencias ambientales. Revista Ecosistemas 13(1): 78-86.

Quero JL. 2006. SADIE como herramienta de cuantificación de la heterogeneidad espacial: casos prácticos en el Parque Nacional de Sierra Nevada (Granada, España). Revista Ecosistemas 15(3): 40-47.

Ramírez JF, E Porcayo. 2009. Estudio de la distribución espacial del muérdago enano (Arceuthobium sp.) en el Nevado de Toluca , México. Madera y Bosques 15(2): 93-112.

Ramírez JF, E Porcayo. 2010. Estudio comparativo de la distribución espacial del muérdago enano (Arceuthobium sp.) en la ladera norte del Parque Nacional Nevado de Toluca, México. Bosque 31(1): 28-38. DOI: 10.4067/S0717$\underline{92002010000100004}$

Ramírez JF, VM Solares, DK Figueroa, JR Sanchez. 2013. Comportamiento espacial de trips (Insecta: Thysanoptera), en Plantaciones comerciales de aguacate (Persea Americana Mill.) en Zitácuaro, Michoacán, México. Acta Zoologica Mexicana 29(3): 545-562.

Ramón BP, M Cruz, MA Zavala. 2016. Factors influencing the dispersion of Arceuthobium oxycedri in Central Spain : evaluation with a new null model for marked point patterns. Forest Pathology 46(6): 1-12. DOI: 10.1111/efp.12279

Ricotta C, LC Grapow, G Avena, C Blasi. 2001. Topological analysis of the spatial distribution of plant species richness across the city of Rome (Italy) with the echelon approach. Landscape and Urban Planning 57(2): 69-76. DOI:
10.1016/S0169-2046(01)00187-6

Rist L, U Shaanker, J Ghazoul. 2011. The Spatial Distribution of Mistletoe in a Southern Indian Tropical Forest at Multiple Scales. Biotropica 43(1): 50-57. DOI: 10.1111/j.17447429.2010.00643.x

Ross CJS. 1987. Maximum likelihood program. Londres, Reino Unido. Rothamsted Experimental Station Harpenden. 95 p.

Rozas V, JJ Camarero. 2005. Técnicas de análisis espacial de patrones de puntos aplicadas en ecología forestal. Investigación Agraria: Sistemas Recursos Forestales 14(1): 79-97.

Samper FJ, J Carrera. 1996. Geoestadística, aplicaciones a la hidrogeología. Barcelona, España. Centro Internacional de Métodos Numéricos en Ingeniería. 484 p.

Sayad E, E Boshkar, S Gholami. 2017. Different role of host and habitat features in determining spatial distribution of mistletoe infection. Forest Ecology and Management 384: 323330. DOI: 10.1016/j.foreco.2016.11.012

SEDEMA (Secretaria del Medio Ambiente, MX). 2010. Integración de Sistemas de Gestión para las Áreas verdes del Distrito Federal mediante un Sistema de Información Geográfica. Gobierno del Distrito Federal. México DF, México. Lemus. 120 p.

Silva AS, TA Mota, NY Pineyro, MG Fernandes, FF Pereira. 2016. Distribución espacial de Vatiga spp. (Hemiptera: Tingidae) en el cultivo de yuca. Acta Biológica Colombiana 21(1):195-200. DOI: 10.15446/abc.v21n1.46762

Steel RG, JH Torrie, JM Castaño. 1985. Bioestadística: principios y procedimientos (Vol. 2). Bogotá, Colombia. McGraw-Hill. 622 p.

Trujillo GM. 2010. Distribución de Poisson. Consultado 08 nov. 2017. Disponible en http://hdl.handle.net/10251/8216

Wang C, R Liu, J Shi, C Martínez. 2015. Traveling waves of a mutualistic model of mistletoes and birds. Dynamical Systems 35(4) 1743-1765. DOI: 10.3934/dcds.2015.35.1743

Ward MJ, DC Paton. 2007. Predicting mistletoe seed shadow and patterns of seed rain from movements of the mistletoebird, Dicaeum hirundinaceum. Austral Ecology 32(2): 113-121. DOI: 10.1111/j.1442-9993.2006.01668.x

Yunuen A, Z Hernández, V Manuel, C Alcalá, L López. 2015. Identificación de daños en el arbolado de tres parques del Distrito Federal. Identification of tree damages of three parks of Mexico City. Revista Mexicana de Ciencias Forestales 6(32): 63-82. 